Ann. Biol. anim. Bioch. Biophys., I968, 8 (2), 29I-299.

\title{
VACCINATION AVEC LA LEUCOCIDINE DANS LA MAMMITE STAPHYLOCOCCIQUE DE LA BREBIS
}

\author{
G. VIDAL \\ avec la collaboration technique de G. BEZARD \\ Station de Pathologie de la Reproduction, \\ Centre de Recherches vétérinaires et zootechniques de Tours-l'Orfrasière, 37 - Nouzilly \\ Institut national de la Recherche agronomique
}

SOMMAIRE

La protection conférée par la leucocidine staphylococcique adsorbée sur phosphate d'alumine a été étudiée sur la brebis. L'étude de cette protection a été faite par comparaison avec des lots témoins et vaccinés par des anatoxines $\alpha-\beta$, en estimant la gravité des mammites expérimentales et en mesurant la croissance des Staphylocoques in vi

Une protection partielle a été obtenue avec les deux vaccins. Les titrages d'anticorps ont montré que les deux vaccins augmentaient, à la fois, les titres anti- $\alpha$ et anti-leucocidine et que, par conséquent, ces vaccins n'étaient pas suffisamment purifiés pour permettre une interprétation précise du rôle de chaque anticorps dans la protection contre l'infection.

\section{INTRODUCTION}

En associant leucocidine, coagulase et hémolysine $\alpha$ dans un vaccin antistaphylococcique, DERBYSHIRE et HELLIWEL L (I962) ont obtenu chez la chèvre un certain degré de protection contre la mammite expérimentale. Une expérience analogue réalisée dans notre laboratoire, en I964, avec un mélange d'anatoxines $\alpha$ et $\beta$, de la coagulase et de la leucocidine brute, nous avait suggéré que 1'anticorps anti-leucocidine jouait un rôle important dans la protection contre l'infection expérimentale de la brebis (résultats non publiés).

Par ailleurs, Plommet en I960, Le GaI, et Plommet en I965, ont montré l'importance du rôle de la réaction leucocytaire dans l'évolution de la mammite chez la brebis. Ils pensaient qu'un vaccin efficace devrait protéger les leucocytes 
contre l'agression par les leucocidines. Cette hypothèse trouvait un appui dans les résultats de MUDD et al. (Ig65) et de SoučkovÁ-ŠTÉPÁnovÁ et al. (I965). Les premiers montrèrent que 1'état des malades atteints d'ostéomyélite chronique était très amélioré par vaccination avec l'anatoxine leucocidine associée, soit à un lysat bactériophagique de staphylocoques, soit à l'anatoxine $\alpha$, soit aux deux à la fois. Les seconds trouvèrent que la vaccination avec de l'anatoxine leucocidine seule, conférait une protection chez le lapin.

Dans le travail présent, nous avons comparé l'activité : ${ }^{\circ}{ }^{\circ}, d^{\prime} u n$ vaccin leucocidine fabriqué au laboratoire, $2^{\circ}$ : d'un vaccin commercial contenant les anatoxines $\alpha$ et $\beta$, parallèlement à un lot témoin non vacciné. L'expérience a montré que le vaccin leucocidine, n'était pas entièrement exempt de toxine $\alpha$ et que le vaccin commercial contenait d'assez fortes quantités de leucocidine. De ce fait, les résultats ne sont pas aussi nets que nous 1'espérions. Cepenđant, l'analyse des cas individuels apporte une forte présomption en faveur d'une protection effective conférée par les anticorps anti-leucocidine.

\section{MATÉRIEL ET MÉTHODES}

\section{Schéma de l'expérience}

Vingt-six brebis, de race Préalpes, en première ou deuxième lactation, ont été réparties en trois lots de huit et neuf animaux, par tirage au sort. Le troupeau était relativement homogène, puisque les animaux étaient indemnes d'infection au moment de l'expérience et que les histogrammes de fréquence des titres d'anticorps avant vaccination montraient une distribution sensiblement normale. Quelques brebis avaient souffert dans les mois précédents d'infections staphylococciques diverses: ce qui explique certains titres d'anticorps un peu élevés avant vaccination.

Les brebis du premier lot ont été vaccinées avec le vaccin anatoxines $\alpha-\beta$; celles du deuxième lot avec le vaccin leucocidine; celles du troisième lot restèrent comme témoins.

\section{Vaccins et vaccinations}

a) Vaccin anatoxines $\alpha-\beta$. - Ce vaccin, provenant de l'Institut Pasteur, est composé d'un mélange d'anatoxines $\alpha$ et $\beta$, dont le titre floculant est de io $U \alpha$ et de $5 \mathrm{U} \beta$ par $\mathrm{ml}$. Ce vaccin est adsorbé sur un gel d'alumine, dans la proportion de ro p. I0o (p/v), au moment de l'emploi (Plommet et LE Gall, ig63). Pour cela, le gel est lavé avec de l'eau physiologique à $8,5 \mathrm{p}$. I 000 et stérilisé à $120^{\circ} \mathrm{C}$ pendant 20 minutes, puis ajouté au mélange d'anatoxines en quantité voulue.

Le protocole de vaccination consiste en trois injections sous-cutanées, faites à une semaine d'intervalle, aux doses de $5^{\circ} \mathrm{U} \alpha$ et $25 \mathrm{U} \beta$, adsorbées sur I $g$ de gel d'alumine, sous un volume de $6 \mathrm{ml}$., pour les deux premières injections, et de Io० $\mathrm{U} \alpha$ et $5 \circ \mathrm{U} \beta$ adsorbées sur $2 \mathrm{~g}$ de gel d'alumine, sous un volume de $12 \mathrm{ml}$ pour la troisième.

b) Vaccin leucocidine. - La leucocidine, produite au laboratoire (VIDAL, 1967) est partiellement purifiée par chromatographie sur C. M. Cellulose selon la méthode de WoodIN (I96i), qui permet la séparation des deux fractions $\mathrm{F}$ et $\mathrm{S}$. Le vaccin est préparé par adsorption des deux fractions séparément sur gel de phosphate d'alumine. Ce gel est préparé à partir de sulfate d'alumine et de phosphate disodique, puis lavé avec un tampon phosphate $\mathrm{O}, \mathrm{I} \mathrm{M}$ à $\mathrm{pH}=7,0$ et stérilisé à $\mathrm{I} 20^{\circ} \mathrm{C}$ pendant 20 minutes.

La vaccination a consisté en 4 injections sous-cutanées, à une semaine d'intervalle, à doses croissantes :

$-5^{\cdot} \mathrm{I}^{4}$ (Dose Minimale Leucotoxique) DMLe de fraction $\mathrm{F}+5^{\cdot} \mathrm{I}^{4} \mathrm{DMLe}$ de fraction $\mathrm{S}$, adsorbées sur $35 \mathrm{mg}$ de gel de phosphate d'alumine, sous un volume de $\mathbf{I} \mathrm{ml}$.

- I $\cdot 10^{5}$ DMLe de fractions F et S, sur $35 \mathrm{mg}$ de gel, sous I $\mathrm{ml}$.

- $2 \cdot 10^{5}$ DMLe des deux fractions, sur $70 \mathrm{mg}$ de gel, sous $2 \mathrm{ml}$.

$-4 \cdot 10^{5}$ DMLe sur $140 \mathrm{mg}$ de gel, sous $4 \mathrm{ml}$. 
c) Date des vaccinations. - La demière injection de vaccin a été faite deux semaines avant la mise à la traite, soit trois semaines avant l'inoculation.

\section{Titrages}

\section{a) Titrage de la leucocidine.}

La méthode est celle décrite par VIDAL (I967). Pour titrer F, on fait une dilution logarithmique de la fraction $F$, à laquelle on ajoute une quantité constante, en excès, de fraction $S$, puis une quantité constante de leucocytes de lapin. L'action de la toxine sur les globules blancs est observée par la réduction de Phénol-Indo-2-6-Dichlorophénol, rajouté après les leucocytes (WooDIN, 1959).

Pour titrer $\mathrm{S}$, on ajoute à la dilution de $\mathrm{S}$ une quantité constante, en excès, de fraction $\mathrm{F}$.

L'unité de titrage est la Dose Minimale Leucotoxique (DMLe). La Dose Test (L $\left.\mathbf{L}^{+}\right)$a été choisie en appliquant l'équivalence établie par Gladstone et VAN Heyningen (I957), soit 500 DMLe pour $\mathrm{I}^{+*}$.

\section{b) Titrage des anticorps anti-leucocidine.}

La méthode est celle de Woodin ( 1961 ), légèrement modifiée. Les sérums, décomplémentés à $56^{\circ} \mathrm{C}$ pendant 30 minutes, sont dilués en échelle logarithmique de $\mathrm{I} / \mathbf{2}$ en $\mathrm{I} / \mathbf{2}$.

Titrages anti-F. - A chaque tube de dilution, on ajoute $\mathrm{I}^{+}$de fraction $\mathrm{F}$ diluée en tampon gélatine-phosphate à $\mathrm{pH}=7,2$. Après incubation de 15 minutes au bain-marie à $37^{\circ} \mathrm{C}$, on rajoute une $\mathrm{L}^{+}$de fraction $\mathrm{S}$ et on titre en présence des leucocvtes de lapin. Ce titrage est confirmé par un second, avec une dose ro fois supérieure de fraction $S$ dans chaque tube. Si l'on obtient le même résultat aux deux titrages, on a bien titré anti-F. Le titre obtenu est exprimé en anti- $\mathrm{L}^{+}$, par l'inverse de la dilution.

Titrages anti-S. - Les dilutions de sérums sont incubées à $37^{\circ} \mathrm{C}$ en présence de $\mathrm{I} \mathrm{L}^{+}$de fraction $\mathrm{S}$. Puis on rajoute à chaque dilution une quantité de $\mathrm{F}$ correspondant au titre trouvé précédemment, plus un excès $\left(4 \mathrm{~L}^{+}\right)$. Le nouveau titre obtenu est le titre anti-S. Dans les deux cas, l'unité anti- $\mathrm{L}^{+}$ correspond à la quantité neutralisant 500 DMLe de chaque fraction.

\section{c) Titrage des anticorps anti- $\alpha$ et anti- $\beta$.}

Ces titrages sont réalisés par neutralisation de l'hémolysine selon la modalité décrite par PLoMMET et LE GALL $(1963)$ avec les toxines $\alpha$ et $\beta$ titrées de l'Ínstitut Pasteur. Le titre est exprimé en unités anti-hémolytiques par référence au sérum international.

Les titrages de tous les anticorps ont été effectués sur tous les animaux, avant la vaccination et avant l'inoculation.

\section{Contrôle des brebis, inoculation et contrôle de l'infection}

On a vérifié l'absence d'infection de la mamelle sur chaque brebis, par des examens bactériologiques et cellulaires, pratiqués sur trois échantillons de lait prélevés à 8 jours d'intervalle et la veille de l'inoculation.

L'inoculation, faite 8 jours après la mise à la traite, $56 \pm 3$ jours après l'agnelage et après la traite du soir, a consisté en l'injection dans un trayon de i I à I 35 germes dans o,2 $\mathrm{ml}$ de suspension de la souche de staphylocoque $\mathrm{n}^{\circ}$ 8I-26, selon la technique de PLOMMET (I960).

Des prélèvements de lait ont été faits 16 heures, $20 \mathrm{~h}, 24 \mathrm{~h}$, et $36 \mathrm{~h}$ après l'inoculation, sur lesquels on a procédé à des numérations :

I. de staphylocoques par ensemencement sur boîtes de gélose au sang de mouton ;

2. de cellules inflammatoires par étalement sur lame de Breed, selon la méthode de LE GaLL et Plommet (1965). Le degré de gravité des mammites a été déterminé 48 heures après l'inoculation, en utilisant l'échelle proposée par Le GaLl et Plommet (I965).

(*) Gladstone et Van Heyningen (I957), proposèrent un système d'unités tel que I $\mathrm{I}^{+}$de leucocidine correspondait à 500 DMLe. C'est cette correspondance que nous avons adoptée dans l'expérience présente. Puis, Woodin (I959) démontre que la leucocidine est en réalité composée de deux fractions $F$ et $S$, agissant en synergie. Pour les titrer, il utilise un sérum auquel il assigne arbitrairement les valeurs de $240 \mathrm{U}$ anti-F et $240 \mathrm{U}$ anti-S. En I 62 , GLADSTONE et al., modifient les unités anti-S de WoodiN en assignant au sérum un titre de $48 \mathrm{U}$ anti-S, au lieu de $240 \mathrm{U}$; ils conservent le titre anti-F (240 U). Les titres, exprimés dans notre système, par rapport au sérum de l'Institut Pasteur titré par GLADS'TONE et al. (1962) donnent l'équivalence suivante, I L+ GLADSTONE correspond à, respectivement $\mathbf{I}, 28 \mathrm{~L}^{+} \mathrm{F}$ et $0,8 \mathrm{~L}^{+} \mathrm{S}$ de notre système. 
G. VIDAL

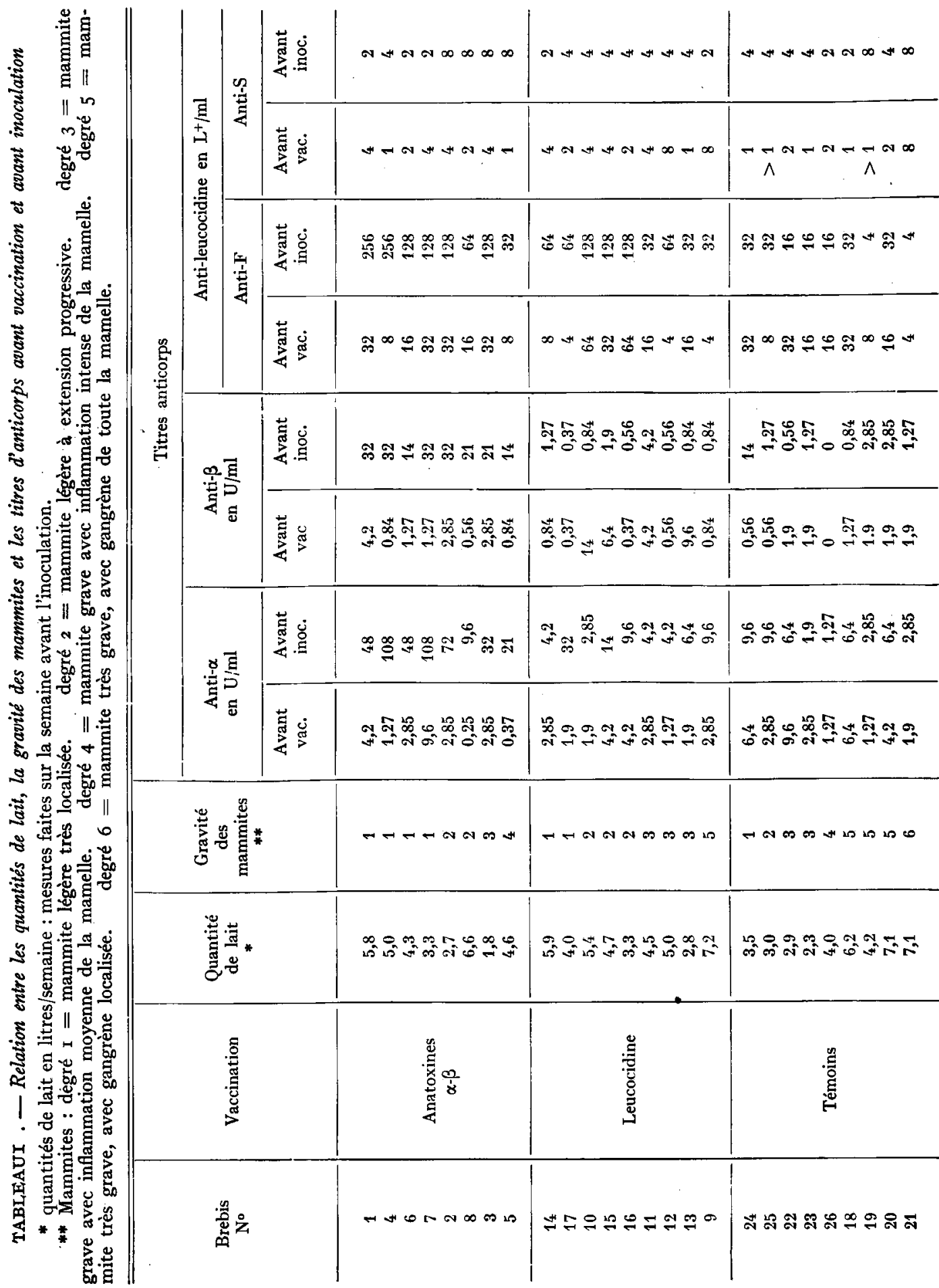




\section{RÉSULTATS}

Les résultats de l'expérience sont consignés dans le tableau r. Nous y avons porté les quantités de lait produites par çhaque brebis (en litres par semaine, mesurées sur la semaine précédant l'inoculation), le degré de gravité des mammites et les titres d'anticorps de tous les animaux, avant vaccination et avant inoculation.

\section{Action de la vaccination sur les titres anticorps}

L'action de la vaccination sur les titres anticorps a été exprimée en pourcentages d'efficacité relative, pour chacun des vaccins utilisés (tabl. 2).

TABLEAU 2

Efficacité relative en $p .100$ des deux vaccins, calculée sur la totalité des animaux de chaque groupe et pour chaque type d'anticorps

\begin{tabular}{|c|c|c|}
\hline Vaccination & Anticorps & $\begin{array}{c}\text { Efficacité relative } \\
(\%)\end{array}$ \\
\hline Anatoxines $\alpha-\beta$ & $\begin{array}{l}\text { Anti- } \alpha \\
\text { Anti- } \beta \\
\text { Anti-F } \\
\text { Anti-S }\end{array}$ & $\begin{array}{r}1638 \\
930 \\
560 \\
76\end{array}$ \\
\hline Leucocidine & $\begin{array}{l}\text { Anti- } \alpha \\
\text { Anti- } \beta \\
\text { Anti-F } \\
\text { Anti-S }\end{array}$ & $\begin{array}{r}200 \\
0 \\
250 \\
18\end{array}$ \\
\hline
\end{tabular}

\section{Action des vaccinations sur la gravité des infactions}

Les vaccins expérimentés n'empêchent pas que l'infection se déclare, mais diminuent le degré de gravité des mammites. L'histogramme des fréquences de gravité (fig. I), montre, que par rapport aux animaux-témoins, chez lesquels la majorité

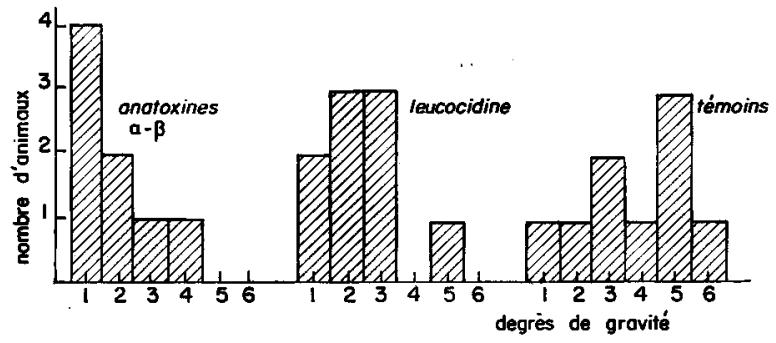

FIG. I. - Histogramme de fréquence des gravités des mammites pour chaque mode de vaccination 
des animaux est atteinte de mammites de degré 5 , on observe un déplacement du mode des histogrammes vers les degrés 2 et 3 pour le vaccin leucocidine et vers le degré I pour le vaccin anatoxines $\alpha-\beta$. La moyenne des gravités pour chaque lot d'animaux exprime différemment et confirme ce résultat $: 2,12$ chez les brebis vaccinées avec les anatoxines $\alpha-\beta, 2,44$ pour les brebis vaccinées avec la leucocidine et 4,0 pour les brebis-témoins.

L'analyse individuelle des cas de mammites chez les animaux du groupe leucocidine montre que, dans certains cas, l'efficacité du vaccin leucocidine est très vraisemblable (tabl. $x$ ). En effet, les mammites bénignes des brebis $n^{\circ}$ Io et ${ }_{4} 4$ ne peuvent guère s'expliquer que par une protection due à la présence d'anticorps anti-F en quantité élevée, car les anticorps anti- $\alpha$ et anti- $\beta$ sont en quantité trop faible pour permettre cette protection. De même en va-t-il pour les brebis n ${ }^{0}$ II, I2 et I3 qui ont

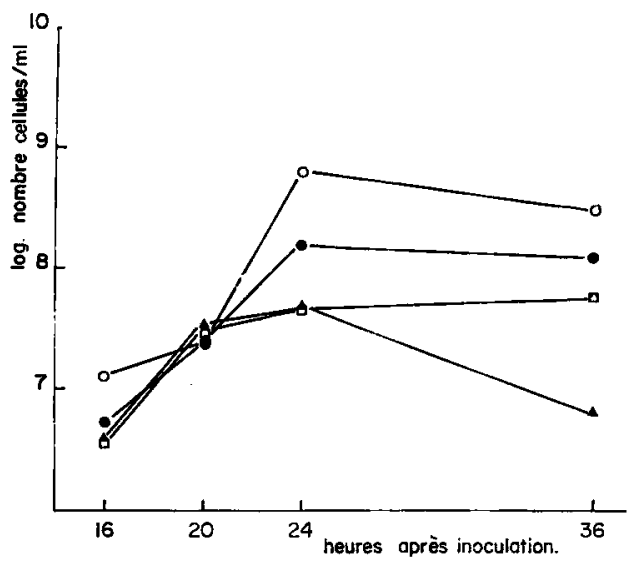

FIG. 2. - Evolution moyenne du nombre $(\log / \mathrm{ml})$ de germes et du nombre $(\mathrm{log} / \mathrm{ml})$ de leucocyles au cours de l'infection

$\square-\square$ : Moyenne des courbes Leucocytes des 26 brebis.

o- - : Moyenne des courbes Staphylocoques des 9 brebis-témoins.

-1: Moyenne des courbes Staphylocoques des 8 brebis vaccinées $\alpha-\beta$

$\bullet-1$ : Moyenne des courbes Staphylocoques des 9 brebis vaccinées Leucocidine.

toutes présenté une mammite de degré 3 , avec des titres anti- $\alpha$ et anti- $\beta$ faibles, mais des titres anti-F relativement forts. Pour les brebis $\mathrm{n}^{0} \mathrm{I} 5$, I6 et $\mathrm{I} 7$ la protection relative observée peut être aussi bien attribuée à la présence d'anticorps anti-F (I28 U, I28 U, $64 \mathrm{U}$, respectivement) qu'aux anticorps anti- $\alpha$ ( $14 \mathrm{U}, 9,6 \mathrm{U}$, et $32 \mathrm{U}$ respectivement). La mammite grave de la brebis $n^{\circ} 9$ (degré 5) s'explique par le fait que sa production laitière était très élevée, puisque comme l'ont montré PLOMMET et RICORDEAU (I960), une forte production de lait est l'un des éléments déterminants de la gravité des mammites. Les brebis mauvaises laitières présentent généralement des mammites de faible gravité. Dans cette expérience, $1 \mathrm{a}$ brebis $\mathrm{n}^{0} 3$ présente une exception à cette règle, puisque cette brebis était la plus mauvaise laitière du troupeau ( $r, 8$ litre/semaine) et que ses titres anti- $\alpha$, anti- $\beta$, anti- $F$ étaient relativement élevés et qu'elle a tout de même été atteinte d'une mammite de moyenne gravité (degré 3). On pouvait s'attendre à obtenir une mammite de degré $I$. 
$L$ 'analyse individuelle des brebis du lot vacciné avec les anatoxines $\alpha-\beta$ ne permet pas de faire la part de protection qui revient à chacun des anticorps $\alpha, \beta$ et leucocidine, car les titres de ces anticorps sont, dans ce lot, très élevés.

\section{Action des vaccinations sur le processus infectieux}

Dans la figure 2, nous avons tracé les courbes moyennes du nombre des staphylocoques en fonction du temps, pour chaque lot, témoin et vaccinés. Le nombre des leucocytes dans les mêmes prélèvements est figuré dans une seule courbe, moyenne de la totalité des animaux : il n'y a, en effet, pas de différence systématique entre les groupes.

On voit l'influence nette de la vaccination sur le nombre de germes; ceci représente vraisemblablement l'efficacité plus ou moins grande de la phagocytose, selon la présence d'anticorps anti-leucotoxiques et anti-toxiques, comme l'ont suggéré LE Gali, et Plommet (I965).

\section{DISCUSSION}

L'augmentation du titre de l'anticorps anti-F de la leucocidine, chez les brebis vaccinées avec les anatoxines $\alpha$ et $\beta$ de 1'Institut Pasteur (tab1. I) montre que ce vaccin contient de la leucocidine et plus particulièrement de la fraction $F$ en assez grande quantité. Le même résultat apparaît dans le tableau $2:$ ce vaccin anatoxines $\alpha-\beta$ présente un pourcentage d'efficacité relative de $560 \mathrm{p}$. Ioo pour la fraction $F$ et de 76 p. Ioo pour la fraction $S$, soit bien plus que notre vaccin leucocidine. Nous avons titré dans le vaccin anatoxines $\alpha-\beta$ son activité leucocidine et son activité anatoxine leucocidine par mesure de son pouvoir de combinaison. Son titre leucocidine est de 3o DMLe $/ \mathrm{ml}$ et son titre anatoxine est de $32 \mathrm{~L}+\mathrm{F} / \mathrm{ml}$ et $2 \times 10^{9} \mathrm{~L}+\mathrm{S} / \mathrm{ml}$. Ceci prouve que la leucocidine traitée par le formol est en partie détoxifiée tout en conservant son pouvoir antigénique. Ceci confirme les résultats de GLADSTONE (I965) et suggère que la leucocidine sous forme d'anatoxine est autant ou plus antigénique que la leucocidine intacte.

Inversement, notre vaccin leucocidine augmente le titre d'antitoxine $\alpha$, avec une efficacité relative de $200 \mathrm{p}$. Ioo (tabl. 2). Ceci nous a révélé la présence de toxine $\alpha$ dans notre leucocidine, à la dose de I U/o,02 $\mathrm{ml}$ dans la fraction $\mathrm{F}$ et I $\mathrm{U} / 0, \mathrm{I} \mathrm{ml}$ dans la fraction S. GLADSTONE and VAN HEYNINGEN (I957), avaient montré que la souche $V 8$, utilisée pour la production de la leucocidine, donnait un peu de toxine $\alpha$. Nous avions décelé, à plusieurs reprises, des traces de cette toxine dans nos surnageants de culture. Il semble bien que la toxine $\alpha$ se concentre par les mêmes voies que celles utilisées pour la purification partielle de la leucocidine.

Les titres anti-S ne sont jamais très élevés (tabl. I), ni dans le lot $\alpha-\beta$,ni dans le lot leucocidine. Le pourcentage d'efficacité relative sur le titre anti-S est très faible, comparé à celui des autres titres anticorps : $76 \mathrm{p}$. roo d'augmentation pour le lot $\alpha-\beta$ et $I 8$ p. Ioo pour le lot leucocidine (tabl. 2 ). Ceci montre que la fraction $S$ est peu antigénique et on peut penser que les anticorps anti-S entrent pour une part minime 
dans la protection des leucocytes contre la leucocidine. Les leucocytes des brebis vaccinées sont en effet morphologiquement intacts et leur phagocytose active (fig. 2), malgré des titres anti-S très bas. Dans ces conditions, une vaccination avec la frac-tion $F$ seule, devrait suffire pour conférer une protection contre la leucocidine. Nos résultats concordent avec les chiffres publiés par MudD et al. (I965) chez l'homme, qui montrent que les titres anti-S augmentent faiblement après vaccination.

LE GALL et PlOMMET (I965) émettaient 1'hypothèse, à 1'examen de leurs courbes de leucocytose, que la vaccination contre les leucocidines, au sens large du terme; devrait conférer une immunité totale sans même qu'il soit nécessaire de vacciner avec les anatoxines $\alpha$ et $\beta$. L'expérience présente infirme cette hypothèse, puisqu'une vaccination contre la plus leucotoxique des leucocidines associée à un vaccin contre les toxines $\alpha$ et $\beta$, dans le vaccin commercial de l'Institut Pasteur, n'a pu permettre d'obtenir cette immunité totale.

La présente expérience ne permet pas de conclure de façon définitive en faveur d'une efficacité relative de la vaccination contre la leucocidine, mais apporte cependant des présomptions sérieuses en faveur de cette thèse. L'expérience mérite d'être reprise après purification plus complète de la leucocidine, en particulier après avoir débarrassé les fractions $\mathrm{F}$ et $\mathrm{S}$ de la toxine $\alpha$ contaminante et après transformation de celles-ci en anatoxines.

Reçu pour publication en décembre 1967.

\section{REMERCIEMEN'TS}

Je remercie M. le Docteur Pillet, de l'Institut Pasteur de Garches, pour m'avoir donné le vaccin anatoxines $\alpha-\beta$. ainsi que les sérums titrés anti- $\alpha$ et anti- $\beta$.

\section{SUMMARY}

VACCINATION WITH LEUCOCIDIN AGAINST STAPHYLOCOCCAL MASTITIS IN EWE

Twenty-six ewes I) not vaccinated, 2) Vaccinated with staphyloloccal toxoids, $\alpha$ and $\beta, 3$ ) Vac. cinated with leucocidin ( $\mathrm{F}$ and $\mathrm{S}$ fractions), were challenged with a virulent strain of Staphylococcus inoculated into the udder.

Severity of mastitis and in vivo growth of staphylococci were compared between the groups and showed a partial protection of vaccinated animals with either of the two vaccins.

Toxoids vaccins increased on average the anti-leucocidin titers, and leucocidin increased slightly anti- $\alpha$ antibodies, showing incomplete purification of the toxins. Thus, it was difficult to understand the role of each antitoxin. However, analysis of results on individual basis suggests that anti-F antibody may have an important role in protection against experimental infection.

\section{RÉFÉRENCES BIBLIOGRAPHIQUES}

Derbyshire J. B., Helliwell B. I., I962. Immunity to experimental staphylococcal mastitis in Goat produced by $\alpha$-lysin, coagulase and leucocidin. Res. Vet. Sci., 3, 56-62.

Gladstone G. P., 1965. Staphylococcal leucocidin toxoid. Brit. J. Exp. Path., 46, 292-307.

Gladstone G. P., MudD S., Hochstein H. D., LEnhart N. A., I 962. The assay of anti-staphylococcal leucocidal components (F and S) in human serum. Brit. J. Exp. Palh., 43, 295-312. 
Gladstone G. P., Van Heyningen W. E., i957. Staphylococcal Leucocidins. Brit. J. Exp. Path., 38, 123-137.

Le Gall A., Plommet M., I965. Observations sur la croissance des staphylocoques et la réaction leucocytaire au cours des premières heures de la mammite expérimentale de la Brebis. Ann. Biol. anim. Bioch. Biophys., 5, II $3^{-1} 3$ o.

Mudd S., Gladstone G. P., Lenhart N. A., 1965. The antigenicity in man of staphylococcal leucocidin toxoid, with notes on therapeutic immunization in chronic osteomyelitis. Brit. J. Exp. Path., 46, 455-472.

PLommet M., 1960. Mammite staphylococcique de la Brebis. Infection expérimentale. Ann. Inst. Pasteur, 88, $439-455$

Plommet M., Le Gall A., 1963. Mammite staphylococcique de la Brebis. III. Recherches sur l'immunité antitoxique et antimicrobienne. Ann. Inst. Pasteur, 104, 779-796.

Plommet M., Ricordeau G., Ig60. Mammite staphylococcique de la Brebis. Influence des modes de traite et de sevrage, du nombre d'agneaux, du stade de lactation et de la production laitière sur le déclenchement de l'infection. Ann. Zootech., 8, 225-240.

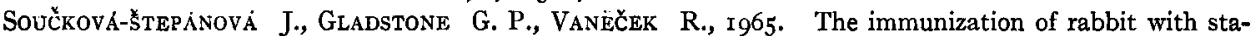
phylococcal leucocidin toxoid, with a report on the pathological histology of their internal organs. Brit. J. Exp. Path., 46, 384-407.

VIDAL G., 1967. Production de la leucocidine de Staphylococcus pyogenes. Ann. Biol. anim. Bioch. Biophys., 7, $209-212$.

Woodin A. M., 1959. Fractionation of a Leucocidin from Staphylococcus aureus. Biochem. J., 73, 225-237.

Woodrn A. M., 196r. Assay of the two components of staphylococcal leucocidin and their antibodies. J. Path. Bact., 81, $63-68$. 